\title{
The sound of size \\ Crossmodal binding in pitch-size synesthesia: A combined TMS, EEG and psychophysics study
}

\author{
Nina Bien ${ }^{*, 1}$, Sanne ten Oever ${ }^{1}$, Rainer Goebel, Alexander T. Sack \\ Department of Cognitive Neuroscience, Faculty of Psychology and Neuroscience, Maastricht University, The Netherlands
}

\section{A R T I C L E I N F O}

\section{Article history:}

Received 2 March 2011

Revised 25 May 2011

Accepted 28 June 2011

Available online 20 July 2011

\section{Keywords:}

Crossmodal

Synesthesia spectrum

Parietal cortex

TMS

ERP

\begin{abstract}
A B S T R A C T
Crossmodal binding usually relies on bottom-up stimulus characteristics such as spatial and temporal correspondence. However, in case of ambiguity the brain has to decide whether to combine or segregate sensory inputs. We hypothesise that widespread, subtle forms of synesthesia provide crossmodal mapping patterns which underlie and influence multisensory perception. Our aim was to investigate if such a mechanism plays a role in the case of pitch-size stimulus combinations. Using a combination of psychophysics and ERPs, we could show that despite violations of spatial correspondence, the brain specifically integrates certain stimulus combinations which are congruent with respect to our hypothesis of pitch-size synesthesia, thereby impairing performance on an auditory spatial localisation task (Ventriloquist effect). Subsequently, we perturbed this process by functionally disrupting a brain area known for its role in multisensory processes, the right intraparietal sulcus, and observed how the Ventriloquist effect was abolished, thereby increasing behavioural performance. Correlating behavioural, TMS and ERP results, we could retrace the origin of the synesthestic pitch-size mappings to a right intraparietal involvement around $250 \mathrm{~ms}$. The results of this combined psychophysics, TMS and ERP study provide evidence for shifting the current viewpoint on synesthesia more towards synesthesia being at the extremity of a spectrum of normal, adaptive perceptual processes, entailing close interplay between the different sensory systems. Our results support this spectrum view of synesthesia by demonstrating that its neural basis crucially depends on normal multisensory processes.
\end{abstract}

(c) 2011 Elsevier Inc. All rights reserved.

\section{Introduction}

In daily life, perception of objects and scenes is rarely unisensory in nature. In fact, any given percept often stimulates multiple senses at the same time, most commonly the visual and auditory modalities. In a world which is highly complex in both the visual and auditory domain, accurately combining or segregating these simultaneous sensory inputs from different modalities is one of the crucial processes in making sense of the world surrounding us. The key function of multisensory integration is to dissociate between stimuli originating from different sources, which should be treated as separate perceptual objects, and stimuli originating from a single source, which should subsequently be perceived and treated as a single object of perception. This raises the question when and how multimodal inputs are combined into a single percept. In contrast to unisensory neurons found in sensory cortices, there are also brain regions containing multisensory neurons, which

\footnotetext{
* Corresponding author at: Department of Cognitive Neuroscience, Faculty of Psychology and Neuroscience, Maastricht University, P.O. Box 616, 6200 MD Maastricht, The Netherlands. Fax: + 31433884125

E-mail address: nina.bien@maastrichtuniversity.nl (N. Bien).

${ }^{1}$ Contributed equally to the study.
}

have spatially overlapping receptive fields in two or more modalities. Different sensory inputs are integrated if they simultaneously activate both receptive fields of a multisensory neuron, thus if they are temporally and spatially congruent (Wallace et al., 1996). Neurons with such multisensory properties have been localised in deep layers of the primate superior colliculus (Wallace et al., 1996). In humans, cortical areas displaying similar characteristics were found in the superior temporal cortex (van Atteveldt et al., 2004) and in the parietal cortex (e.g. Calvert, 2001; Calvert et al., 2000, 2001).

In case of sensory inputs which are ambiguous with regard to one of the criteria of spatial and temporal correspondence, the brain has to decide whether or not to integrate these inputs into a single percept. Under these circumstances, top-down cognitive mechanisms may overrule bottom-up differences between temporal onset and/or spatial origin of the different sensory inputs. Of course some top-down couplings could be attributed to repeated exposure to different sensory counterparts of an event, being perceived together constantly over and over again, until an overlearned semantic link is formed. This might be how we learn that a dog barks, whereas a cat meows-and not the other way around. This overlearned pairing could possibly exert top-down influence on the binding process in case of bottom-up ambiguity. However, repeatedly perceiving some stimuli in accordance with each 
other cannot explain why different days have different colours to different people (Simner et al., 2006), why white balls squeak (Mondloch and Maurer, 2004), why small is bright and big is dark (Cohen Kadosh et al., 2007), why small numbers are positioned in the left half of mental space (Dehaene et al., 1993)-in short, why some stimuli, which are at first glance not obviously related, are consistently linked by multisensory mechanisms which are thought to be operating from a bottom-up perspective. Even to a level at which multisensory integration actually deteriorates performance (e.g. Driver, 1996; McGurk and MacDonald, 1976; Parise and Spence, 2009).

Apparently, certain automatic crossmodal mapping patterns occur in the normal population, yet bear semblance of the rare phenomenon of synesthesia. While synesthesia literally means "joining of the senses", it is commonly referred to as a sensory perception in a modality additional to the one which was physically stimulated (see e.g. Hubbard et al., 2005). Although it has always fascinated the general public, scientifically synesthesia represents a rather underexposed perceptual phenomenon. There is growing consensus that the research of synesthesia could advance our understanding of the normal and abnormal human brain and cognition (e.g. Cohen Kadosh and Henik, 2007; Mulvenna and Walsh, 2006). Extreme, rather rare forms of synesthesia like grapheme-colour synesthesia have traditionally received most scientific attention. This leads to a view of synesthetic perception, and its underlying mechanisms, as being qualitatively different from normal multisensory integration. However, examples of systematic crossmodal mapping which cannot be explained simply by bottom-up stimulus characteristics could be interpreted as milder forms of synesthesia (Martino and Marks, 2001), which are not just rare anomalies, but rather adaptive and widespread traits in the normal population. One example is that of the "squeaking white balls", described by Mondloch and Maurer (2004). They observed young children consistently appointing a small sized or light coloured ball as the source of a high pitched tone, and vice versa. There is increasing evidence that many of such common mapping patterns between e.g. pitch, size, colour and distance (Cohen Kadosh et al., 2008; Parise and Spence, 2008; Romei et al., 2009) are retained into adulthood. For example, one widespread form of synesthesia is the mental number line, a spatial distribution of small and large numerical values across horizontal mental space (Dehaene et al., 1993) which can modulate spatial attention (Fischer et al., 2003).

Besides such crossmodal mappings, which seem to rely on certain top-down binding characteristics, reports of synesthesia and crossmodal integration sharing common neural mechanisms also hint at a non-qualitative distinction. For example, TMS disruption of parietal cortex disrupts automatic integration of synesthetic mappings between graphemes and colours in grapheme-colour synesthetes (Esterman et al., 2006; Muggleton et al., 2007). Parietal cortex has also been reported in other studies into synesthesia (Beeli et al., 2008; Rouw and Scholte, 2010; Weiss et al., 2005). Interestingly, the same brain region is known for its role in multisensory integration (Calvert et al., 2001; Werner and Noppeney, 2010).

Moreover, whereas synesthetic mappings were initially considered random, coincidental associations, it turns out that there are recurrent patterns across individuals with regard to, for example, grapheme-colour associations (Brang et al., 2011; Eagleman, 2010; Rich et al., 2005), and across languages and alphabets (Brang et al., 2011; Cohen Kadosh et al., 2007; Eagleman, 2010; Kim, 2010). Similar synesthetic mapping patterns have also been reported across synesthetes and non-synesthetes (Spector and Maurer, 2008; Ward et al., 2006). Apparently, there are shared underlying representations for at least certain synesthetic patterns. The purpose of such shared mapping patterns has yet to be explored, however it is likely that they are to some extent meaningful and advantageous.

In addition, there is the well-described difference between socalled 'associator' versus 'projector' synesthetes with regard to their qualitative experiences, and the way synesthesia influences their performance on psychophysical tasks (Dixon et al., 2004; Hubbard and Ramachandran, 2005; Muggleton et al., 2007; Rouw and Scholte, 2010). Projectors' sensory byproducts interfere stronger with performance on a synesthetic Stroop task than the associations reported by associator synesthetes (Dixon et al., 2004). It appears that synesthesia is not an all-or-nothing condition, as it has traditionally been considered, but contrarily there is variability with regard to how much synesthesia a synesthete experiences, and consequently, to what degree their synesthesia modulates automatic perceptual processes.

As a result, viewpoints have recently been shifting more towards synesthesia being at the extremity of a spectrum of normal, adaptive perceptual processes, entailing close interplay between the different sensory systems (Eagleman, 2009; Esterman et al., 2006; Martino and Marks, 2001; Mulvenna and Walsh, 2006; Nikolic, 2010; Sagiv and Ward, 2006). Consequently, the concept and the criterion of what is termed synesthesia might need to be expanded (Nikolic, 2010). In line with this emerging spectrum view of synesthesia, we propose that synesthesia and crossmodal integration are indeed closely linked. In fact, we suggest that when simple criteria such as temporal and spatial congruency fail to explain why sensory stimuli are integrated or not, synesthetic processes become of relevance by providing intrinsic mappings which allow for top-down influence on the integration process. Synesthesia as such can be seen as a crossmodal process which relies on neural structures shared with normal crossmodal processing, which can manifest itself in different strengths, exerting graded influence on perception as well as on modulation of automatic processes, which entails shared and possibly meaningful mapping patterns across individuals, and which has many milder, widespread manifestations in the normal population. The spectrum view of synesthesia meets all these criteria, with common, prevalent instances of crossmodal mapping as the 'missing link' between normal multisensory integration and extreme forms of synesthesia. Although even extreme forms of synesthesia have been found to adhere to certain rules or regularities, it is most likely the variety of common, widespread forms which can inform us best on yet unacknowledged crossmodal processes which are clearly underlying and influencing everyday perception.

In the current study we tried to deepen our understanding of common synesthetic mappings in the normal population. To this end, we employed the Ventriloquist paradigm (Driver, 1996): when presented with spatially segregated but temporally and semantically congruent audiovisual speech, the brain decides that because sound and vision are simultaneous and congruent, they must belong to the same source. Since human visual perception is more accurate in the spatial domain than auditory perception, the spatial origin of the speech sound is misallocated to the location of the lip movements, leading the brain to believe that visual and auditory speech originated from the same spatial source. A similar ambiguous situation arises when sensory stimuli belonging to multiple sensory events arrive simultaneously from approximately the same location. Again, the brain has to decide whether or not to integrate some of these stimuli, and which ones belong together. The Ventriloquist illusion, in line with other ambiguous multisensory settings in which the brain decides to integrate sensory events despite violation of the basic rules of correspondence (e.g. McGurk and MacDonald, 1976), or vice versa, raises the question which type of information is of crucial importance. Clearly, basic bottom-up stimulus characteristics such as temporal and spatial correspondence are not always sufficient to explain the 'when' and 'when not' of multisensory integration.

This paradigm allowed us to systematically probe the multisensory system by deliberately violating basic bottom-up rules of integration and enabled us to systematically and quantitatively verify whether small sized objects and high pitches are grouped together (henceforth labelled "synesthetically congruent"), as compared to small objects and low pitches, in randomly selected volunteers. If a small object and a high pitch are indeed considered highly semantically congruent, the 
brain would group them together notwithstanding the spatial separation between them, which would result in decreased accuracy in determining the spatial origin of the tone with regard to the visual stimulus. In a next step, we verified whether multisensory integration plays a crucial role in binding these precepts, by using repetitive transcranial magnetic stimulation (TMS) in order to temporarily disrupt the right intraparietal cortex, a brain region previously reported of relevance for both synesthetic and crossmodal binding processes. During task execution we recorded brain activity with 64 EEG-electrodes to provide a detailed temporal overview of neural processes reflecting synesthetic mapping, as well as to assess the neurophysiological correlate underlying TMS-induced changes in synesthetic behaviour. This combination of psychophysics, ERP and TMS revealed significant interactions between synesthesia-mediated behaviour and ongoing neuronal processes in higher perceptual brain areas within frontal and parietal cortex.

\section{Materials and methods}

\section{Participants}

A total of 14 neurologically healthy volunteers with normal or corrected to normal vision (aged between 18 and 25), 4 of which were male, participated in this study. Participants were randomly selected, and none of the participants reported to be synesthetic. Eleven of these participants simultaneously underwent EEG recording. All participants were unaware of the goal of the study until after having completed their participation. Before the start of each experimental session, each participant provided written informed consent and was screened for TMS experimentation safety by an independent medical supervisor. Ethical approval was given by the local medical ethical committee. Participants were rewarded with student participation credits.

\section{Paradigm and psychophysics procedure}

Each experimental participation consisted of two sessions, separated by at least one day. The first session consisted of two parts: a tailoring procedure was carried out before the actual experimental session commenced.

The tailoring procedure at the start of the first session was carried out to ensure that the difficulty level of the experimental task was similar for each participant, regardless of inter-individual differences. Participants were asked to fixate at the centre of a screen, and indicate via a button press whether they thought a tone originated from a location left or right of fixation. Their individual auditory localising threshold was determined using a staircase procedure. During this tailoring procedure, participants were comfortably seated with their head in a chin rest, at $55 \mathrm{~cm}$ viewing distance from the computer screen.

After the tailoring procedure, EEG preparation and (depending on the session) TMS application were finalised, the actual experimental session started. Again, participants were asked to fixate at the centre of the screen. In each trial, a visual circle stimulus appeared left or right of fixation, while concurrently a pure tone auditory stimulus was emitted from two speakers flanking the screen. Both stimuli lasted $200 \mathrm{~ms}$ and differed in spatial location. The visual stimulus could be either large or small, and the tone could have a low or a high pitch (Fig. 1). The combination of visual size and tone pitch could be either congruent according to the hypothesis of pitch-size synesthetic mappings in the normal population (large stimulus-low pitched tone or small stimulus-high pitched tone), or incongruent (large stimulus-high pitched tone or small stimulus-low pitched tone). Participants were asked to make a forced-choice judgment on the spatial origin of the tone, either left (right index finger response) or

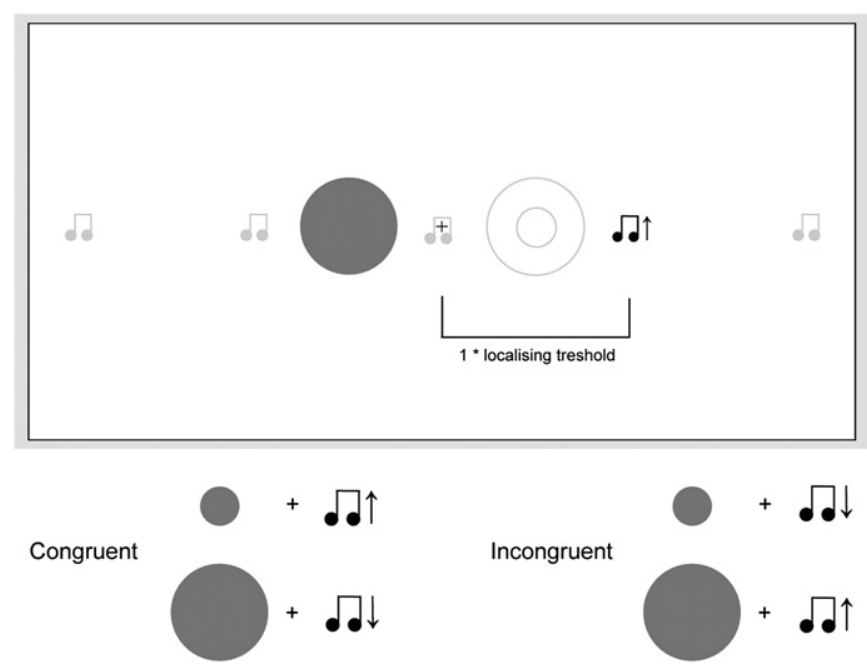

Fig. 1. Pitch-size ventriloquist paradigm. Simultaneously with the visual presentation of a circle with a diameter of either $5.2^{\circ}$ (large) or $2.1^{\circ}$ (small) of visual angle, a high $(4500 \mathrm{~Hz})$ or low $(250 \mathrm{~Hz})$ tone was presented at one out of five possible locations. Participants indicated whether the tone originated from the left or, as in the example shown, from the right of the visual stimulus. Visual and auditory stimulus could either be congruent (small-high or large-low) or incongruent (small-low or large-high) according to the hypothesis of pitch-size synesthesia. Stimulus locations were tailored according to individual auditory localising thresholds.

right (right middle finger response) of the visual stimulus. Accuracy and reaction times of the responses were recorded.

The experiment consisted of 320 trials per session divided over four blocks of approximately $4 \mathrm{~min}$ each. Trial onset asynchrony was randomly jittered between 3 and $4 \mathrm{~s}$. Condition order was randomised, with the restriction that an equal amount of trials was presented in each condition throughout the whole study. Hence the number of trials in each condition was fully balanced. A 2 by 2 factorial design was employed, with the conditions Synesthetic Congruency (congruent or incongruent) and TMS (TMS or no TMS) as the two within-subject factors.

\section{Stimulus material}

Auditory stimuli consisted of high $(4500 \mathrm{~Hz}$ at $63 \mathrm{~dB})$ or low ( $250 \mathrm{~Hz}$ at $72 \mathrm{~dB}$ ) pitched pure tones, lasting for $200 \mathrm{~ms}$. Sound levels were chosen in accordance with equal loudness curves (Suzuki and Takeshima, 2004), assuring that the perceived loudness for the high and low pitched tones was equal. The sounds were presented by two loudspeakers placed on both sides of the computer screen. By systematically varying the ratio between the loudness produced by each of the two speakers, five illusory auditory locations were created on a horizontal axis (Fig. 1). These locations were determined according to each participant's individual auditory localising threshold, as detected in a staircase tailoring procedure. Auditory stimuli were situated left or right of fixation at one or two times of the individual threshold distance. The fifth location was the centre of the screen itself. To increase difficulty, and thus maximise multisensory integration processes (Meredith and Stein, 1986; Stein et al., 2009), white noise with an intensity of $65 \mathrm{~dB}$ was presented in the background during the entire experimental session.

Visual stimuli consisted of a large $\left(5.2^{\circ}\right.$ visual angle $)$ or small $\left(2.1^{\circ}\right.$ visual angle) $20 \%$ grey circle, presented also for $200 \mathrm{~ms}$ on a 17" TFT screen (Samsung SyncMaster $931 \mathrm{DF}$ ) on white background. Visual stimuli were presented at distances 0.5 times the individual auditory localising threshold left or right of fixation. Throughout the experiment a $20 \%$ grey fixation cross was shown in the centre of the screen. 
Presentation software (Neurobehavioral Systems, Inc., Albany, NY) was used for both stimulus presentation and recording of the behavioural responses.

\section{TMS design and apparatus}

During one of the two sessions, the order of which was randomised between participants, continuous theta burst TMS (Huang et al., 2005) was administered over the right parietal P4 EEG electrode position (10-20 EEG positioning system), which has been shown to overlie intraparietal sulcus (see e.g. Hilgetag et al., 2001). Biphasic magnetic stimulation was generated using a Magstim Rapid ${ }^{2}$ stimulator (The Magstim Company, Whitland, UK). Magnetic pulses were delivered with a hand-held figure of eight coil $(70 \mathrm{~mm}$ standard coil, The Magstim Company, Whitland, UK) placed tangentially to the scalp with the handle pointing $45^{\circ}$ in the lateral-inferior direction.

Continuous theta burst TMS is an inhibitory patterned TMS protocol, applied over the course of $40 \mathrm{~s}$, with an affectivity outlasting the stimulation itself by approximately $1 \mathrm{~h}$ (Huang et al., 2005). Application of TMS is inevitably accompanied with specific side effects. The clicking sounds as well as the sensations on the scalp can be distracting during behavioural task execution, and delivering the magnetic pulse distorts ongoing EEG recording. Employing offline theta burst TMS allows for TMS artefact-free recording of behavioural as well as EEG data. Stimulation was delivered at $80 \%$ of the individual resting motor threshold (with a maximum not exceeding $45 \%$ of the maximum output of the stimulator). Effectively, participants were stimulated on average at $70.8 \%$ of their individual resting motor threshold. To ensure that the offline effects of TMS did not affect behaviour during the no TMS condition, sessions were at least two days apart.

\section{EEG apparatus and data acquisition}

ERPs were recorded via a $64 \mathrm{Ag}-\mathrm{AgCl}$ electrodes BrainCap MR EEG cap (BrainProducts GmBh, Munich, Germany) and a BrainAmp MR Plus EEG amplifier (BrainProducts $\mathrm{GmbH}$, Munich, Germany) with a sampling rate of $1000 \mathrm{~Hz}$. Online EEG recording was performed with BrainVision Recorder (BrainProducts $\mathrm{GmBh}$, Munich, Germany). All recordings were referenced online to the $\mathrm{Cz}$ electrode. Grounding was provided by a separate electrode located posterior-centrally on the head. The vertical electro-oculogram (VEOG) was recorded from electrodes placed above and below the left eye. EEG preparation was completed before TMS was applied and lasted approximately $1 \mathrm{~h}$. All electrodes were inserted with Abralyt 2000 electrolyte gel (Easycap, Germany) and subsequently fiddled with a small wooden stick until impedance was below $50 \mathrm{k} \Omega$.

\section{Behavioural and TMS data analysis}

Outliers and trials with a reaction time below $200 \mathrm{~ms}$ post stimulus presentation were discarded from further analysis. Trials were labelled as synesthetically congruent or incongruent based on the hypothesis of pitch-size synesthetic mappings. In order to first verify whether pitch-size synesthesia was indeed a common trait within our sample of non-synesthetic participants, mean values of accuracy per participant, per condition, were taken from the session without TMS and entered into a Repeated Measures ANOVA procedure with the within-subject factor Synesthetic Congruency (synesthetically congruent or incongruent). Subsequently, the effect of TMS on pitch-size synesthesia was evaluated by entering mean values of accuracy per participant, per condition into a two-way Repeated Measures ANOVA procedure with the within-subject factors Synesthetic Congruency (synesthetically congruent or incongruent) and TMS (TMS or no TMS). The same analyses with an additional factor Accuracy (correct or incorrect) was applied to the reaction time data. Alpha values of pair-wise comparisons were Bonferroni corrected to correct for multiple comparisons.

\section{ERP data analysis}

EEG data were pre-processed and analysed using Brain Vision Analyzer 2.0 (Brain products, Munich, Germany). Data was rereferred offline to the algebraic average reference and filtered with a $50-\mathrm{Hz}$ notch filter and band-pass filter $(0.5-70 \mathrm{~Hz}, 12 \mathrm{~dB} / \mathrm{oct})$. Continuous EEG data were divided into epochs ranging from -500 to $1500 \mathrm{~ms}$ relative to stimulus onset. Baseline was corrected using $200 \mathrm{~ms}$ of pre-stimulus activity as a reference. Artefacts were automatically detected and manually checked through visual inspection. Artefacts were removed per individual channel. Subsequently epochs were averaged per condition and combined into a group average for each condition, which was subsequently used for data analysis.

Automatic peak detection was employed in four ERP components of interest: P1 (90-150 ms), N1 (150-250 ms), P2 (200-300 ms) and N3 (250-350 ms). Resulting mean amplitudes within an interval of $2 \mathrm{~ms}$ around each peak were used for further analysis. These amplitudes were collapsed into two spatial clusters of electrodes: frontal (electrodes Fz, F1, F2, F3, F4, AFz, AF3, AF4, AF7, AF8, FPz, FP1, FP2, FC3 and FC4) and parietal (electrodes CP1, CP3, P3, CP5, P5, CP4, CP2, CP6, P4, P6, Pz, P2 and P1). To first establish how pitch-size synesthesia is reflected in brain potentials, without the influence of TMS, the resulting mean amplitudes of the four time bins within these two clusters were subsequently used as dependent variables in a Repeated Measures Multivariate ANOVA (RM MANOVA) with the factor Congruency (congruent or incongruent). Additionally, the effect of right parietal TMS on the observed effects of synesthetic congruency was evaluated using a two-way RM MANOVA with the factors Congruency (congruent or incongruent) and TMS (TMS or no TMS). Post-hoc analyses were conducted for the interaction effect of Congruency per level of TMS.

In order to get more insight into the nature of the interaction between the ERP components, we additionally calculated Pearson product-moment correlation coefficients between the relevant components parietal N1, frontal P2, parietal P2 and frontal N3.

\section{Combined behavioural-ERP analysis}

To determine which ERP component reflects pitch-size synesthesia and its modulation after TMS, we calculated Pearson product-moment correlation coefficients between the four aforementioned ERP components and behavioural accuracy over the four different conditions. Since interactions between the factors Congruency and TMS could not be incorporated in the correlation analysis directly, we computed individual incongruent minus congruent accuracy difference scores for No TMS and TMS separately, and correlated those with the equivalent ERP component amplitude difference scores. Considering that TMS is an experimental manipulation of brain function, this information provides direct information about the neural correlates of observed behavioural effects.

\section{Results}

\section{Behavioural and TMS results}

Two participants showed an opposite behavioural pattern, and were thus discarded from further analyses, with the exception of correlational analyses which rely on different statistical assumptions. In accordance with the hypothesis of pitch-size synesthesia in the normal population, synesthetically congruent trials were defined as those which combined a high pitched tone with a small visual stimulus or a low pitched tone with a large visual stimulus, and synesthetically incongruent trials as 
those which combined a high pitched tone with a large visual stimulus or a low pitched tone with a small visual stimulus. If pitch-size synesthesia is indeed a common trait in the normal population, multisensory integration resulting from the Ventriloquist illusion would decrease the perceived spatial distance between the synesthetically related auditory and visual source in the congruent condition, thereby hampering auditory localisation in synesthetically congruent as compared to synesthetically incongruent trials.

Without TMS, participants were indeed more accurate on synesthetically incongruent trials as compared to congruent trials $\left(\mathrm{F}(1,11)=23.332, \mathrm{p}<.01, \eta \mathrm{p}^{2}=.680\right)$ (Fig. 2A). As hypothesised, judging the spatial origin of the auditory relative to the visual stimulus was more difficult when stimuli were synesthetically congruent according to our hypothesis of pitch-size mappings in the normal population. Hence, automatic integration of synesthetically related audiovisual stimuli hindered an accurate spatial judgment of the auditory stimulus with regard to the visual stimulus. Reaction times did not show any significant effects, ruling out speedaccuracy trade-off accounts for the observed accuracy differences.

Application of TMS to the right parietal cortex significantly interacted with this Synesthetic Congruency effect $(F(1,11)=6.637, p<.05$, $\left.\eta \mathrm{p}^{2}=.376\right)$, and thus crucially changed the observed behavioural difference pattern. While without TMS participants were significantly less accurate in synesthetically congruent trials, this adverse effect of synesthetic congruency was significantly diminished after disruption of right parietal cortex $\left(\mathrm{F}(1,11)=.253, \mathrm{p}=1.00, \eta \mathrm{p}^{2}=.000\right)$ (Fig. 2B). In other words, after right parietal TMS, automatic integration of synesthetic congruent stimuli no longer hampered auditory spatial localisation judgment, supposedly because the automatic, synesthetically induced multimodal integration of these stimuli was prevented. Importantly, TMS did not affect overall accuracy $(F(1,11)=.000$, $\mathrm{p}=.987, \eta \mathrm{p}^{2}=.000$ ), discarding nonspecific TMS effects as an account for the observed phenomenon.

\section{ERP results}

Firstly, by evaluating simple effects of Synesthetic Congruency without TMS, we investigated how pitch-size synesthesia was embodied by event-related brain potentials. Although the overall multivariate effect of Synesthetic Congruency was not significant in a RM MANOVA without TMS $\left(\mathrm{F}(1,9)=7.585, \mathrm{p}=.274, \eta \mathrm{p}^{2}=.984\right)$, univariate analyses showed a significant effect of synesthetic congruency within the parietal P2 component around $250 \mathrm{~ms}$ after stimulus onset $(\mathrm{F}(1,9)=5.626$, $\mathrm{p}<.05, \eta \mathrm{p}^{2}=.413$ ). Synesthetic congruency resulted in increased ERP amplitudes. Other components were not significantly modulated by synesthetic congruency. Hence, specifically the P2 component at parietal recording sites was significantly influenced by the integration of the synesthetic congruent stimuli, showing an increased positivity during congruent as compared to incongruent trials.

Subsequently, we tested how application of TMS to the right parietal cortex influenced the multisensory integration of pitch-size stimuli in event-related brain potentials. When the factor TMS was entered into the RM MANOVA, an overall significant interaction effect was revealed between Synesthetic Congruency and TMS $(F(1,9)=360.671, p<.05$, $\left.\eta \mathrm{p}^{2}=1.000\right)$. Separate univariate analyses over mean amplitudes per electrode cluster (parietal and frontal) per component (P1, N1, P2 and N3), revealed that TMS most strongly modulated the P2 component recorded from parietal sites $\left(\mathrm{F}(1,9)=12.147, \mathrm{p}<.01, \eta \mathrm{p}^{2}=.603\right)$. Increased positivity during synesthetically congruent as compared to incongruent trials was reduced after right parietal disruption with TMS (Figs. 3 and 4). In addition, a very similar interaction between Synesthetic Congruency and TMS was observed in the N3 component recorded frontally around $300 \mathrm{~ms}$ after stimulus onset $(\mathrm{F}(1,9)=6.249$, $\mathrm{p}<.05, \eta \mathrm{p}^{2}=.439$ ) (Fig. 3). A pattern of increased negativity during synesthetically congruent compared to incongruent trials was again reduced after TMS. Hence, disruption of right parietal cortex with TMS significantly influenced brain potentials recorded from parietal sites around $250 \mathrm{~ms}$, and from frontal sites around $300 \mathrm{~ms}$. The initially increased amplitude as a result of multisensory integration was diminished following right parietal TMS.

Besides this interaction effect, subsequent univariate analyses indicated a trend towards a Synesthetic Congruency effect in the N1 component at parietal recording sites around $200 \mathrm{~ms}$ after stimulus onset $\left(\mathrm{F}(1,9)=4.744, \mathrm{p}=.061 \eta \mathrm{p}^{2}=.372\right)$, with higher amplitudes in synesthetically incongruent compared to congruent trials. In addition, P2 recorded from frontal sites revealed a significant Synesthetic Congruency effect $\left(\mathrm{F}(1,9)=7.645, \mathrm{p}<.05, \eta \mathrm{p}^{2}=.489\right)$, consisting of an increased positivity during incongruent compared to congruent trials. The different amplitudes during synesthetically congruent and incongruent trials indicate that these two early components are associated with the automatic synesthetic integration, but in contrast to parietal P2 and frontal N3, they are not influenced by magnetic disruption of right parietal cortex. They are thus not reflecting the observed behavioural TMS-induced modulations. As the N1 component was not significant, but only indicated a trend, the link with pitch-size synesthesia is possibly also weaker than the significant parietal P2 component.

The similar interaction patterns between Synesthetic Congruency and TMS observed in parietal P2 and frontal N3 imply that these components are closely linked, possibly even originating from a single
A

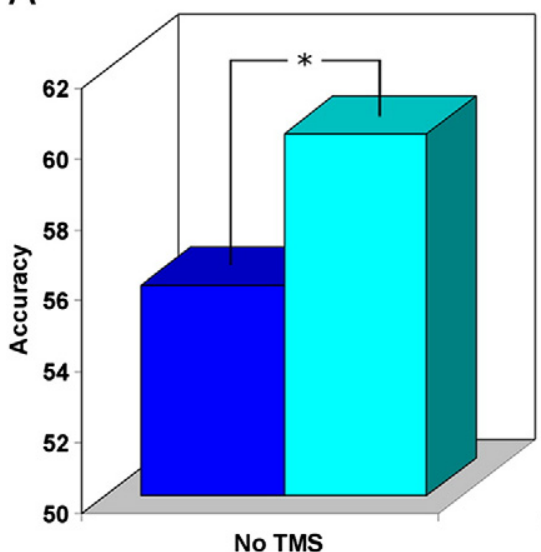

B

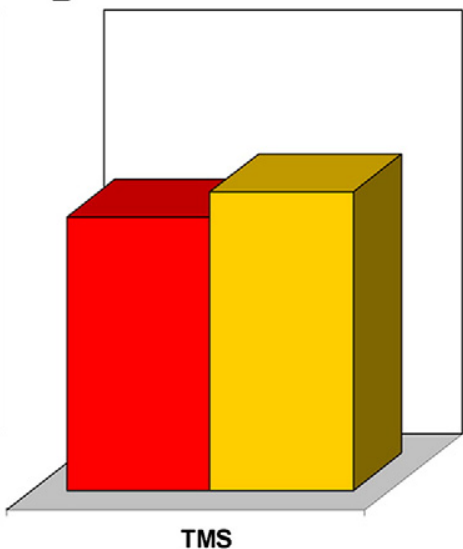

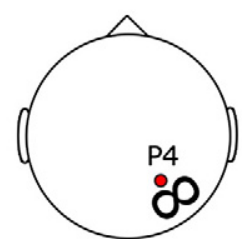

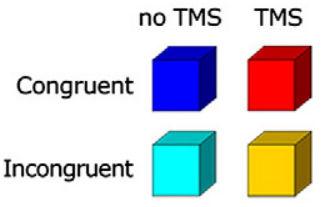

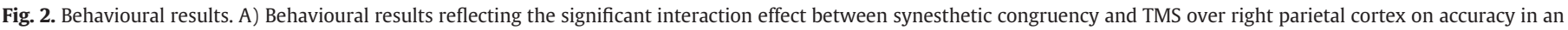

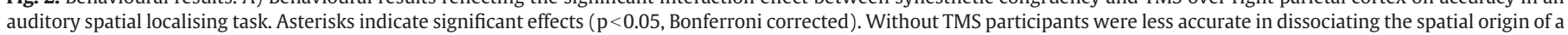
tone when visual stimulus and tone were synesthetically congruent. After TMS this difference disappeared almost completely. 

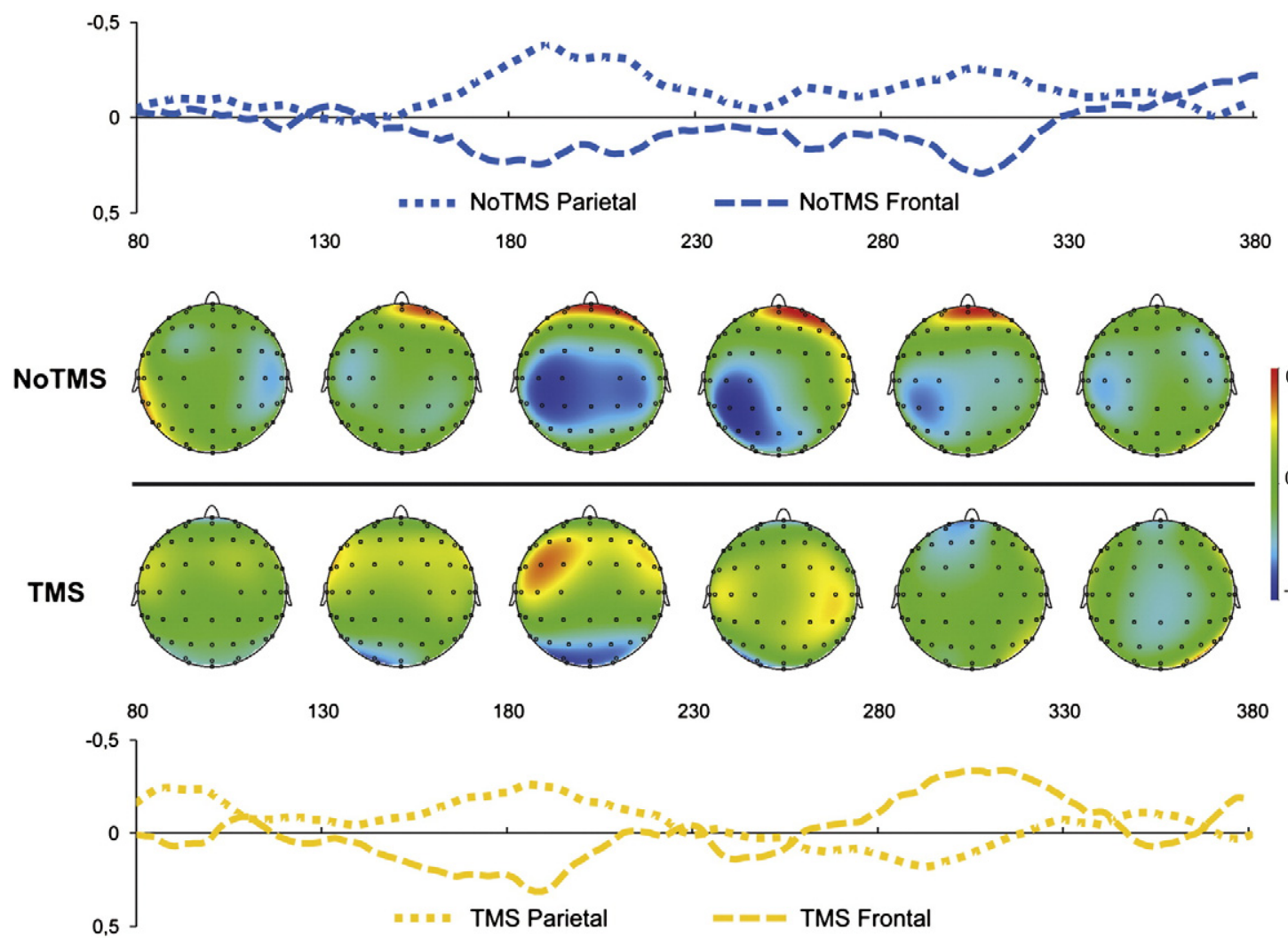

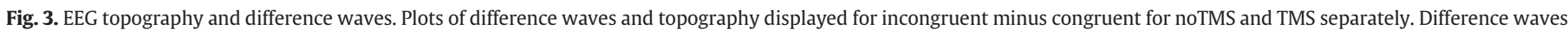

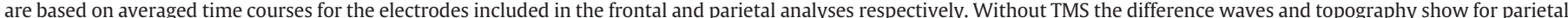

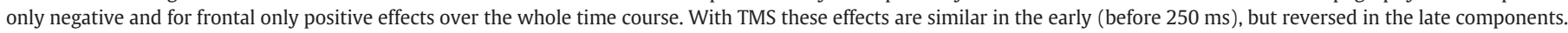

dipole. The same could be suggested for the Synesthetic Congruency main effects in parietal N1 and frontal P2. Indeed, Pearson productmoment analysis showed significant correlations between Parietal P2 and frontal N3 across conditions (Table 1A and Fig. 5). This suggests that the parietofrontal P2-N3 is associated with the initial boost of multisensory integration caused by synesthetic congruency (as reflected in the reduced behavioural accuracy), and with the disruption of this integration by parietal TMS stimulation. Similarly, parietal N1 and frontal P2 were significantly correlated across conditions (Table 1A), implying a parietofrontal N1-P2 complex. Most likely, this complex also relates to synesthetic congruency, reflecting an earlier processing stage which was not modulated by TMS stimulation.

\section{Correlations between behavioural and ERP results}

Because TMS is an experimental manipulation of ongoing cortical processing, resulting modulations of both behaviour and event-related brain potentials can be causally attributed to the application of TMS. Following from this, we can hypothesise that certain TMS-induced changes in behaviour are reflected by certain TMS-induced ERP modulations, allowing us to make direct inferences about the neural basis of a certain behavioural phenomenon. As described above, behavioural performance as well as the parietal P2 ERP component showed significant modulations as a result of synesthetic congruency, and were both significantly modulated by TMS over right parietal cortex. To confirm that the observed behavioural disruption of synesthetic multisensory integration-and thus pitch-size synesthesia as a whole-was indeed reflected in parietal P2, we computed Pearson product-moment correlation coefficients between behavioural performance and the four relevant ERP components. Indeed, a significant correlation was only revealed between behavioural performance and the parietal P2 component (Table 1B and Fig. 5). Without TMS, there was a trend towards a negative correlation effect between Congruency and behavioural performance $(\mathrm{r}=-.583, \mathrm{n}=11, \mathrm{p}=.060)$. After right parietal TMS, a significant positive correlation was observed $(r=.648$, $\mathrm{n}=11, \mathrm{p}<.05)$, suggesting that only the parietal P2 component, emerging around $250 \mathrm{~ms}$, is functionally linked to the automatic integration of synesthetically congruent pitch-size stimuli.

\section{Discussion}

The current study investigated multisensory pitch-size associations, using a combination of psychophysics, transcranial magnetic stimulation and event-related potentials. Multisensory perception, or the process of accurately integrating or segregating incoming sensory events according to their mutual or separate origin, is usually governed by bottom-up stimulus characteristics, such as spatial and temporal correspondence of incoming sensory inputs. However, in case of ambiguity with regard to these bottom-up characteristics, the brain has to decide whether two stimuli belong to the same event, or not. We hypothesise that widespread, subtle forms of synesthesia provide crossmodal mapping patterns which underlie and influence multisensory perception on a large scale, but which especially become apparent when bottom-up features provide insufficient or contradictory information. Our aim was to investigate if such a mechanism plays a role in the case of pitch-size stimulus combinations. To this end we employed a pitch-size variant of the spatial Ventriloquist illusion. By introducing this paradigm we could test whether pitchsize synesthesia introduces ambiguity into the multisensory process, and subsequently observe how the brain deals with this synesthetically induced paradoxical situation. Our results support the emerging 

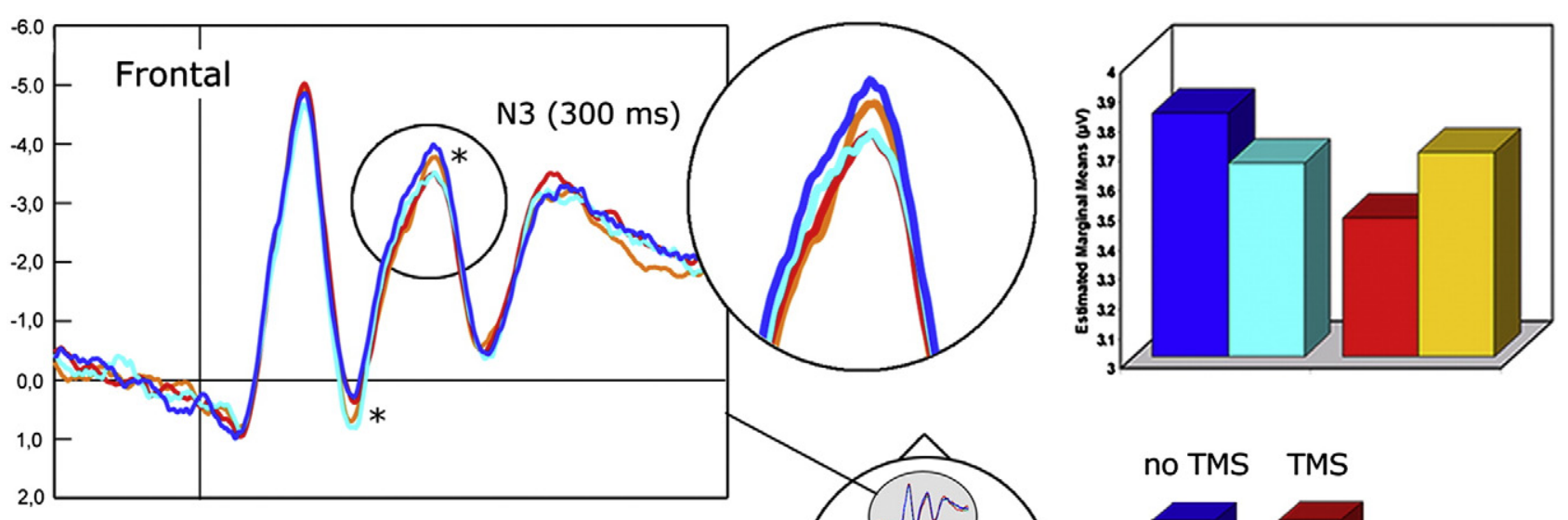

no TMS TMS
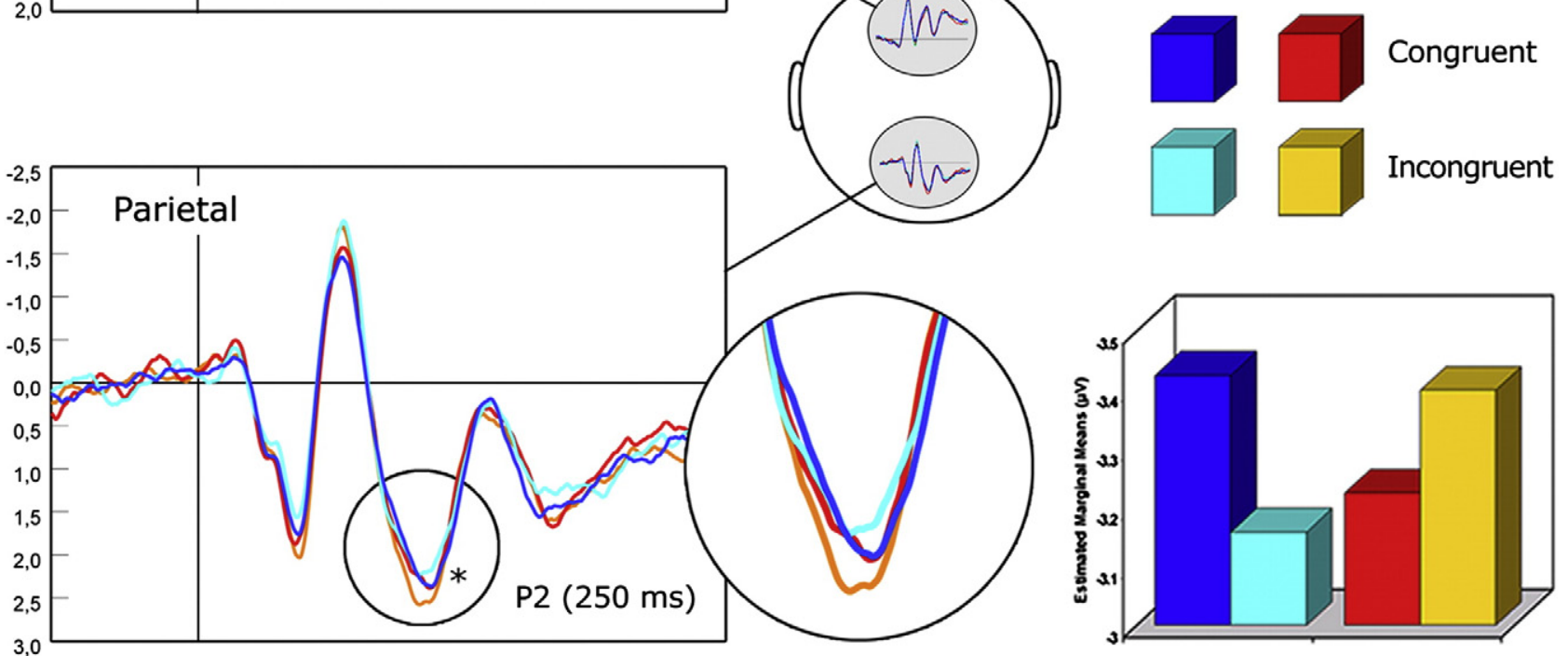

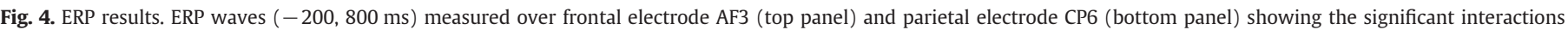

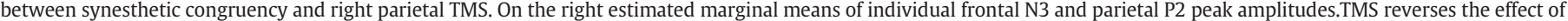
synesthetic congruency.

Table 1

Correlations between ERP components and behaviour.

\begin{tabular}{|c|c|c|c|c|c|}
\hline A & & & N1 parietal & P2 parietal & P2 frontal \\
\hline \multirow[t]{6}{*}{ No TMS } & Congruent & P2 parietal & .167 & & \\
\hline & & P2 frontal & $-.928^{* *}$ & -.231 & \\
\hline & & N3 frontal & -.300 & $-.894^{* *}$ & .268 \\
\hline & Incongruent & P2 parietal & .226 & & \\
\hline & & P2 frontal & $-.927^{* *}$ & -.176 & \\
\hline & & N3 frontal & -.331 & $-.876^{* *}$ & .178 \\
\hline \multirow[t]{6}{*}{ TMS } & Congruent & P2 parietal & .445 & & \\
\hline & & P2 frontal & $-.867^{* *}$ & -.506 & \\
\hline & & N3 frontal & .573 & $.659^{*}$ & -.508 \\
\hline & Incongruent & P2 parietal & .490 & & \\
\hline & & P2 frontal & $-.941^{* *}$ & -.431 & \\
\hline & & N3 frontal & -.346 & $-.923^{* *}$ & .259 \\
\hline
\end{tabular}

\begin{tabular}{lllll} 
B & N1 parietal & P2 parietal & P2 frontal & N3 frontal \\
\hline No TMS & -.103 & $-.583^{\mathrm{T}}$ & .062 & .160 \\
TMS & -.495 & $.648^{*}$ & -.119 & -.071
\end{tabular}

A) Within-subject correlations between the mean amplitudes of four relevant ERP components. Single asterisks indicate significance at the 0.05 level (2-sided), double asterisks indicate significance at the 0.01 level (2-sided). Parietal N1 amplitude significantly correlates with frontal P2 amplitude in all conditions. The same is observed for parietal P2 and frontal N3. B) Correlations between behavioural TMS effects and the mean amplitudes of four relevant ERP components. The asterisk indicates significance at the 0.05 level (2-sided), $T$ indicates a strong trend towards an effect $(p=0.06$, 2 -sided). Since the interaction effects cannot be correlated directly, individual mean incongruent minus congruent accuracy scores are correlated with ERPs for no TMS and TMS separately. These results support the notion that the TMS-induced changes in the parietal P2 component directly account for the observed behavioural changes. spectrum view of synesthesia. We argue that multisensory integration and synesthesia are actually part of a continuum, with mild, widespread variants of synesthesia as the 'missing link' between normal crossmodal processes and extreme forms of synesthesia.

In the current study randomly selected healthy volunteers were presented with simultaneous visual and auditory stimuli differing in spatial location, and were asked to make a forced-choice judgment on the spatial origin of the tone. The visual stimulus could be either large or small, and the tone could have a low or a high pitch. Automatically grouping low pitched sounds and large visual objects together would seem like a behaviourally beneficial trait, one that could help to make sense of the world around us, and simplify or speed up some of our daily challenges. Recent studies (Parise and Spence, 2008, 2009) reported some initial proof of the existence of pitch-size synesthesia, using a similar approach. If a process is deeply rooted into behaviour, it will still be carried out if this results in decreased performance. We assumed that if low pitched sounds are indeed matched with large visual stimuli, and vice versa, these synesthetically congruent objects would be grouped together by brain structures involved in multisensory integration, despite the fact that the objects actually originated from different spatial locations. The spatial origin of the sound would be pulled towards the location of the visual stimulus. Judging the spatial origin of the auditory stimulus would become more difficult because of the brain's effort to ascribe the congruent perceptions to a single source. This is exactly what we observed: behavioural performance with regard to judging the spatial origin of a sound 


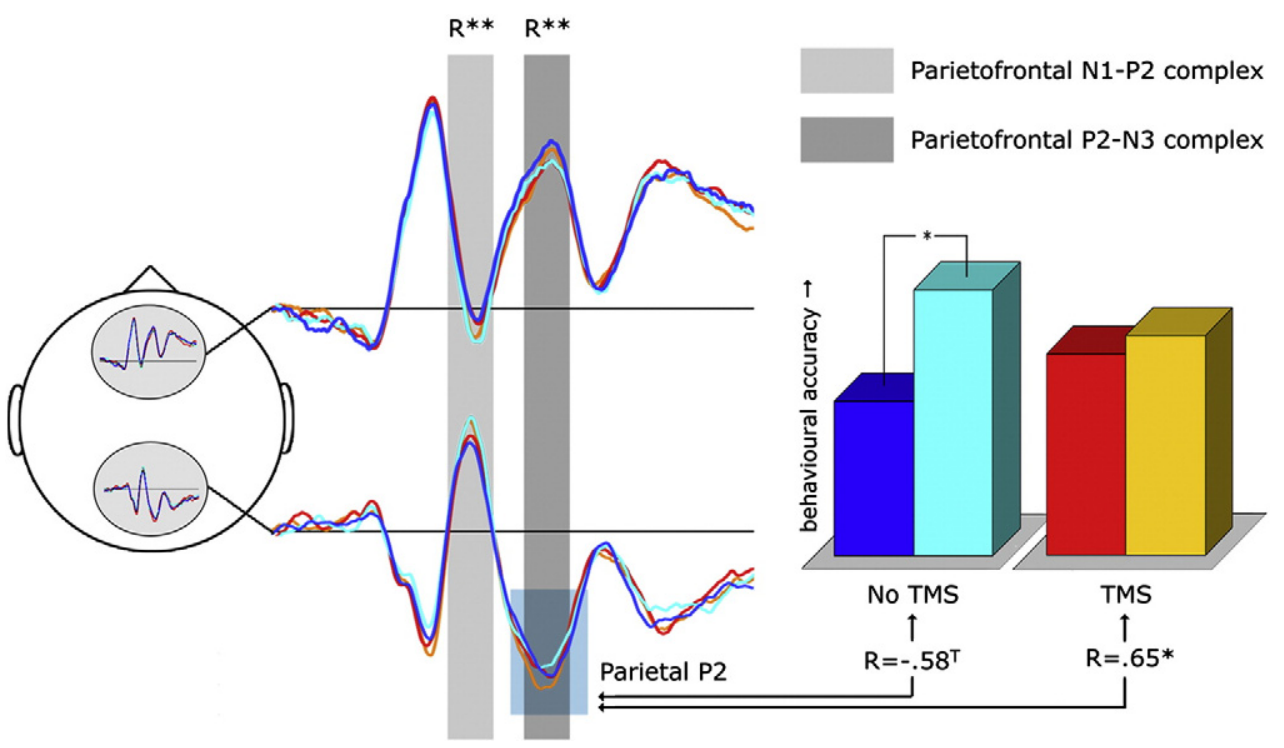

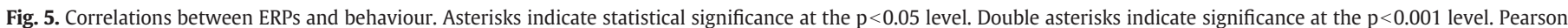

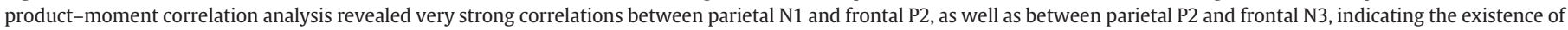

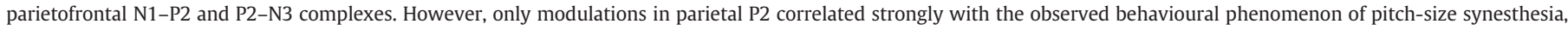

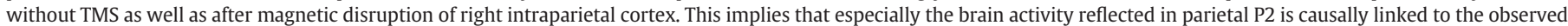

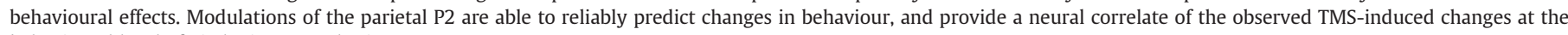
behavioural level of pitch-size synesthesia.

was decreased when sound and visual object were synesthetically congruent. This supports that pitch-size synesthesia is a widespread trait in the normal population. In addition, the fact that this integration occurred even in a situation in which it is actually detrimental to performance, i.e. while the participants were aiming at keeping the stimuli segregated to localise the sound, implies that the process is compulsory and highly automatic.

Based on these psychophysical data we can confirm that sounds with a certain pitch are automatically bound with visual objects with a certain size, even if one of the basic rules of integration, spatial correspondence, is violated. This is an example of crossmodal integration which cannot be explained solely by simple bottom-up features of the stimuli. In contrast, it seems that there is an underlying mapping of pitch-size associations. We argue that this underlying mapping constitutes a subtle form of synesthesia, common in the normal population, and that disruption of this synesthetic process will also inevitably disrupt multisensory integration, or vice versa, because they are qualitatively equivalent. To test this hypothesis, we magnetically disrupted ongoing neuronal processing in the right intraparietal sulcus, a higher perceptual brain area known to be responsible for the integration of congruent multisensory inputs, and observed the consequences on both behaviour and brain potentials. Without TMS, synesthetic congruency resulted in decreased accuracy on auditory spatial judgment. Interestingly, TMS specifically increased performance on those synesthetically congruent trials. This effect can only be explained by disruption of multisensory integration: topdown signals no longer overrule the bottom-up information, thereby wiping out the pitch-size synesthesia, and leaving the auditory locations unchanged and more easily localisable.

Since TMS is an experimental manipulation of brain function, its resulting effects on behaviour and brain potential can provide causal information on brain-behaviour relationships. In this case, we were interested in how the observed behavioural effects of right parietal TMS are reflected in the ongoing brain activity. The differential eventrelated brain potentials with regard to synesthetically congruent and incongruent trials observed in the parietal N1 component, which are significantly correlated with a similar pattern in subsequent frontal P2, could point in the direction of an early synesthetic effect. The increased amplitudes during incongruent trials in the parietofrontal N1-P2 complex might reflect enhanced discriminative or distorted categorization processes (Vogel and Luck, 2000), which speaks to a combined representation of the congruent stimuli already at an early stage of processing. However, since this component only shows an influence of synesthetic congruency, and no influence of TMS, it cannot account for the improved auditory spatial judgment in synesthetically congruent trials after TMS. The low correlation between frontal N1 and behavioural results further supports this notion.

The subsequent P2-N3 complex, on the other hand, shows significant interaction effects between congruency and TMS, and moreover, in a pattern that is comparable with that observed in the behavioural data: as a result of TMS, the difference in brain potential amplitude between congruent and incongruent trials is reduced, even reversed. This effect is first visible in the parietal P2 component around $250 \mathrm{~ms}$, and subsequently in the frontal N3 component around $300 \mathrm{~ms}$. Although significant correlations between parietal P2 and frontal N3 suggest a parietofrontal P2-N3 complex possibly originating from a single dipole, the significant correlation between behavioural results and parietal P2, but not frontal N3, indicates that especially the brain activity reflected in parietal P2 is causally linked to the observed behavioural effects. Apparently, modulations of the parietal P2 are able to reliably predict changes in behaviour. Considering that TMS is an external manipulation of brain function, it seems safe to conclude that TMS over the right intraparietal sulcus diminishes the Ventriloquist illusion arising from synesthetically congruent pitch-size combinations, which is reflected most strongly in the parietal P2 ERP component around $250 \mathrm{~ms}$ after stimulus onset. The sign reversal of the parietal P2 correlation could be explained by a complete reversal of the incongruent-congruent amplitude differences guided only with a decrease of the accuracy differences. Why the patterns do not follow each other is not certain. The reversal might be influenced by sites different from TMS stimulation, since EEG components normally originate from multiple sources.

We can conclude that multisensory integration plays an important role in pitch-size mapping, a widespread and adaptive form of synesthesia. These results support the increasingly cogent view of 
synesthesia as part of a spectrum ranging from normal crossmodal processes, via subtle and prevalent forms of synesthesia, to the curious, more extreme forms that have attracted most scientific attention so far. Currently, two competing theories on the neural substrates of synesthesia are under debate. According to these theories, synesthesia is either ascribed to excess anatomical connections between low-level sensory areas, which are normally pruned in development (Hubbard et al., 2005; Rouw and Scholte, 2007; Weiss et al., 2005), or to a disinhibition of feedback from higher perceptual to unisensory areas (Grossenbacher and Lovelace, 2001; Mulvenna and Walsh, 2006). Until hitherto empirical research on this topic has not been able to clearly dissociate between these two theoretical accounts for the phenomenon of synesthesia. Whereas Cohen Kadosh et al. (2009) were able to induce grapheme-colour synesthesia in otherwise non-synesthetic participants using posthypnotic suggestion, supporting the disinhibited feedback theory, Rouw and Scholte (2007) uncovered increased anatomical connections in synesthetes, which furthermore differed between projectors and associatiors (Rouw and Scholte, 2010). With regard to our current results, the observations that TMS over a higher order, crossmodal region like the right intraparietal sulcus crucially interfered with the synesthetic process, and that ERP data reflect modulations in parietal and subsequently frontal sites at relatively long latencies of around $250 \mathrm{~ms}$, speak more to the disinhibited feedback theory than to the excess anatomical connections theory.

\section{Conclusion}

In conclusion, although pitch-size congruency can be considered an adaptive trait, it hampers performance in an auditory localisation task as a result of the Ventriloquist illusion: synesthetic congruency results in multisensory binding, and consequently auditory sources are misallocated to the visual source. Transcranial magnetic stimulation over right intraparietal sulcus, a known multisensory integration site, is able to wipe out this Ventriloquist effect, and specifically improve performance on synesthetically congruent trials. This experimental manipulation is reflected in the parietal P2 component, which correlates strongly with behavioural performance both with and without TMS. Future studies employing time-resolved TMS around P2-latency could elaborate on the contribution of this component. The current results confirm that pitch-size synesthesia is a common trait within the normal population, and that normal multisensory processes play a crucial role in this process, thus supporting the increasingly cogent spectrum view of synesthesia. A multi-methodological approach, investigating the effects of magnetic disruption of specific cortical processes on both recordings of eventrelated brain potentials and behavioural measures of performance, enabled us to provide detailed information about the cortical components and temporal aspects of this process. However, this in itself does not fully solve the debate about whether synesthesia is an isolated, curious phenomenon, or whether it is part of a continuum shared with normal multisensory processes, with subtle synesthetic processes as the yet unacknowledged missing link. Whether to call these effects 'crossmodal-plus' or 'synesthesia-light' is purely a matter of semantics. We suggest that future studies should aim at further exploring these processes and their interaction with multisensory perception, as well as their link with more extreme forms of synesthesia.

\section{Acknowledgments}

This study was supported by a grant to A.T.S. from the Dutch Organization for Scientific Research (NWO; grant number 452-06003 ) and the Sirius program of the Dutch Ministry of Education, Culture and Science. We thank our medical supervisor Cees van Leeuwen, and our independent physician Martin van Boxtel. We also thank Judith Peeters and Joel Reithler for their assistance with the EEG equipment.

\section{References}

Beeli, G., Esslen, M., et al., 2008. Time course of neural activity correlated with coloredhearing synesthesia. Cereb. Cortex 18, 379-385.

Brang, D., Rouw, R., et al., 2011. Similarly shaped letters evoke similar colors in grapheme-color synesthesia. Neuropsychologia 49, 1355-1358.

Calvert, G.A., 2001. Crossmodal processing in the human brain: insights from functional neuroimaging studies. Cereb. Cortex 11, 1110-1123.

Calvert, G.A., Campbell, R., et al., 2000. Evidence from functional magnetic resonance imaging of crossmodal binding in the human heteromodal cortex. Curr. Biol. 10, 649-657.

Calvert, G.A., Hansen, P.C., et al., 2001. Detection of audio-visual integration sites in humans by application of electrophysiological criteria to the BOLD effect. NeuroImage 14, 427-438.

Cohen Kadosh, R., Henik, A., 2007. Can synaesthesia research inform cognitive science? Trends Cogn. Sci. 11, 177-184.

Cohen Kadosh, R., Henik, A., et al., 2007. Small is bright and big is dark in synaesthesia. Curr. Biol. 17, R834-R835.

Cohen Kadosh, R., Brodsky, W., et al., 2008. Mental representation: what can pitch tell us about the distance effect? Cortex 44, 470-477.

Cohen Kadosh, R., Henik, A., et al., 2009. Induced cross-modal synaesthetic experience without abnormal neuronal connections. Psychol. Sci. 20, 258-265.

Dehaene, S., Bossini, S., et al., 1993. The mental representation of parity and number magnitude. J. Exp. Psychol. Gen. 122, 371-396.

Dixon, M.J., Smilek, D., et al., 2004. Not all synaesthetes are created equal: projector versus associator synaesthetes. Cogn. Affect. Behav. Neurosci. 4, 335-343.

Driver, J., 1996. Enhancement of selective listening by illusory mislocation of speech sounds due to lip-reading. Nature 381, 66-68.

Eagleman, D.M., 2009. The objectification of overlearned sequences: a new view of spatial sequence synesthesia. Cortex 45, 1266-1277.

Eagleman, D.M., 2010. A large-scale analysis of synesthesia reveals clustering of subtypes and influence of grapheme shape on color associations. Annual Meeting of the Society for Neuroscience, San Diego.

Esterman, M., Verstynen, T., et al., 2006. Coming unbound: disrupting automatic integration of synesthetic color and graphemes by transcranial magnetic stimulation of the right parietal lobe. J. Cogn. Neurosci. 18, 1570-1576.

Fischer, M.H., Castel, A.D., et al., 2003. Perceiving numbers causes spatial shifts of attention. Nat. Neurosci. 6, 555-556.

Grossenbacher, P.G., Lovelace, C.T., 2001. Mechanisms of synesthesia: cognitive and physiological constraints. Trends Cogn. Sci. 5, 36-41.

Hilgetag, C.C., Theoret, H., et al., 2001. Enhanced visual spatial attention ipsilateral to rTMS-induced 'virtual lesions' of human parietal cortex. Nat. Neurosci. 4, 953-957.

Huang, Y.Z., Edwards, M.J., et al., 2005. Theta burst stimulation of the human motor cortex. Neuron 45, 201-206.

Hubbard, E.M., Ramachandran, V.S., 2005. Neurocognitive mechanisms of synesthesia. Neuron 48, 509-520.

Hubbard, E.M., Arman, A.C., et al., 2005. Individual differences among grapheme-color synesthetes: brain-behavior correlations. Neuron 45, 975-985.

Kim, C., 2010. Cross-linguistic approaches to color-graphemic synesthesia. Annual Meeting of the Society for Neuroscience, San Diego.

Martino, G., Marks, L.E., 2001. Synesthesia: strong and weak. Curr. Dir. Psychol. Sci. 10, 61.

McGurk, H., MacDonald, J. 1976. Hearing lips and seeing voices. Nature 264, 746-748.

Meredith, M.A., Stein, B.E., 1986. Visual, auditory, and somatosensory convergence on cells in superior colliculus results in multisensory integration. J. Neurophysiol. 56, 640-662.

Mondloch, C.J., Maurer, D., 2004. Do small white balls squeak? Pitch-object correspondences in young children. Cogn. Affect. Behav. Neurosci. 4, 133-136.

Muggleton, N., Tsakanikos, E., et al., 2007. Disruption of synaesthesia following TMS of the right posterior parietal cortex. Neuropsychologia 45, 1582-1585.

Mulvenna, C.M., Walsh, V., 2006. Synaesthesia: supernormal integration? Trends Cogn. Sci. 10, 350-352.

Nikolic, D., 2010. Is synesthesia actually ideaesthesia?: the role of concepts in the phenomenon. Society for Neuroscience, San Diego, CA.

Parise, C., Spence, C., 2008. Synesthetic congruency modulates the temporal ventriloquism effect. Neurosci. Lett. 442, 257-261.

Parise, C.V., Spence, C., 2009. 'When birds of a feather flock together': synesthetic correspondences modulate audiovisual integration in non-synesthetes. PLoS One 4, e5664.

Rich, A.N., Bradshaw, J.L., et al., 2005. A systematic, large-scale study of synaesthesia: implications for the role of early experience in lexical-colour associations. Cognition 98, 53-84.

Romei, V., Murray, M.M., et al., 2009. Preperceptual and stimulus-selective enhancement of low-level human visual cortex excitability by sounds. Curr. Biol. 19, 1799-1805.

Rouw, R., Scholte, H.S., 2007. Increased structural connectivity in grapheme-color synesthesia. Nat. Neurosci. 10, 792-797.

Rouw, R., Scholte, H.S., 2010. Neural basis of individual differences in synesthetic experiences. J. Neurosci. 30, 6205-6213.

Sagiv, N., Ward, J., 2006. Crossmodal interactions: lessons from synesthesia. Prog. Brain Res. 155, 259-271. 
Simner, J., Mulvenna, C., et al., 2006. Synaesthesia: the prevalence of atypical crossmodal experiences. Perception 35, 1024-1033.

Spector, F., Maurer, D., 2008. The colour of Os: naturally biased associations between shape and colour. Percept. Lond. 37, 841.

Stein, B.E., Stanford, T.R., et al., 2009. Challenges in quantifying multisensory integration: alternative criteria, models, and inverse effectiveness. Exp. Brain Res. 198, 113-126.

Suzuki, Y., Takeshima, H., 2004. Equal-loudness-level contours for pure tones. J. Acoust. Soc. Am. 116, 918-1933.

van Atteveldt, N., Formisano, E., et al., 2004. Integration of letters and speech sounds in the human brain. Neuron 43, 271-282.
Vogel, E.K., Luck, S.J., 2000. The visual N1 component as an index of a discrimination process. Psychophysiology 37, 190-203.

Wallace, M.T., Wilkinson, L.K., et al., 1996. Representation and integration of multiple sensory inputs in primate superior colliculus. J. Neurophysiol. 76, 1246-1266.

Ward, J., Huckstep, B., et al., 2006. Sound-colour synaesthesia: to what extent does it use cross-modal mechanisms common to us all? Cortex 42, 264-280.

Weiss, P.H., Zilles, K., et al., 2005. When visual perception causes feeling: enhanced cross-modal processing in grapheme-color synesthesia. Neurolmage $28,859-868$.

Werner, S., Noppeney, U., 2010. Distinct functional contributions of primary sensory and association areas to audiovisual integration in object categorization. J. Neurosci. 30, 2662-2675. 